http://dx.doi.org/10.35381/racji.v6i10.1170

\title{
La pensión vitalicia en la ley orgánica de servicio público en el territorio ecuatoriano
}

The life pension in the organic law of public service in the Ecuadorian territory

\author{
Rosa María Suárez-Barzola \\ dq.rosamsb58@uniandes.ed.ec \\ Universidad Regional Autónoma de los Andes, Quevedo \\ Ecuador \\ https://orcid.org/0000-0002-5144-4950 \\ Wilson Eduardo Castro-Núñez \\ abogadowilsoncastro@gmail.com \\ Universidad Regional Autónoma de los Andes, Quevedo \\ Ecuador \\ https://orcid.org/0000-0002-2500-5145 \\ Pamilys Milagros Moreno-Arvelo \\ uq.pamilysmoreno@uniandes.edu.ec \\ Universidad Regional Autónoma de los Andes, Quevedo \\ Ecuador \\ https://orcid.org/0000-0001-8913-4352
}

Recepción: 11 de septiembre 2020

Revisado: 20 de octubre 2020

Aprobación: 18 de diciembre 2020

Publicación: 01 de enero 2021 


\title{
RESUMEN
}

La investigación tiene por objetivo analizar la pensión vitalicia y su vigencia desde la ley orgánica de servicio público en el territorio ecuatoriano. Se basó desde una centralidad metodológica en un tipo descriptiva documental con diseño bibliográfico. Los resultados alcanzados permitieron concretar la necesidad de cambiar la figura jurídica de pensión vitalicia a pensión de reconocimiento de méritos a ex presidentes y ex vicepresidentes del Ecuador limitando también el pago de este beneficio a 10 años después de haber cumplido su mandato, al considerar que la economía está en depresión al depender en su $80 \%$ o $90 \%$ del desarrollo económico que genere el gobierno para sustentar todos los gastos, por ello que si se limita esta figura jurídica se evitaría quitar una parte del presupuesto del estado y desarrollar otras actividades para el progreso económico del país.

Descriptores: Seguridad social; gasto público; derecho laboral; derecho constitucional. (Palabras tomadas del Tesauro UNESCO).

\begin{abstract}
The objective of the research is to analyze the life pension and its validity from the organic law of public service in the Ecuadorian territory. It was based from a methodological centrality on a descriptive documentary type with bibliographic design. The results achieved made it possible to specify the need to change the legal status from a lifetime pension to a merit recognition pension for former presidents and former vice-presidents of Ecuador, also limiting the payment of this benefit to 10 years after having served their mandate, considering that the The economy is in depression as it depends for its $80 \%$ or $90 \%$ of the economic development generated by the government to support all expenses, so if this legal figure is limited, it would avoid removing a part of the state budget and developing other activities for the economic progress of the country.
\end{abstract}

Descriptors: Social security; public expenditure; labour law; constitutional law. (Words taken from the UNESCO Thesaurus). 


\section{INTRODUCCIÓN}

La pensión vitalicia tiene antecedentes históricos, con el nacimiento de la democracia en el Ecuador, se creó a partir del uno de enero de 1980 mediante decreto ejecutivo firmado el ex presidente por Jaime Roldós Aguilera el pago de pensiones vitalicias a ex presidentes y ex vicepresidentes de la República. Debido al proceso de dolarización que se afianzó en 1998, estas remuneraciones que eran en sucres fueron también dolarizadas. (Jerez-Jimenez, 2018).

El artículo 135 de la (Ley Orgánica de Servicio Público, 2010), establece que Los señores ex presidentes y vicepresidentes, constitucionales de la república, que sean elegidos constitucionalmente por votación popular, tendrán el derecho a una pensión vitalicia mensual, equivalente al setenta y cinco por ciento de la remuneración vigente otorgada para su cargo, exceptuándose de este beneficio a los mandatarios que fueron revocados del mandato.

Durante los últimos años han existido vacíos legales con respecto a exceptuar el cobro a los ex mandatarios que fueron revocados de su mandato, sin embargo, ex mandatarios como Abdalá Bucaram, Jamil Mahuad y Lucio Gutiérrez que fueron derrocados durante su mandato siguen recibiendo su pensión, transgrediendo lo que establece la Ley Orgánica de Servicio Público. (Gomez-Ponce, 2018).

Según el autor (Quizhpe-Benítez, 2019), la palabra vitalicia se refiere a un beneficio que se obtiene hasta el final de la vida, de ahí la naturaleza de las pensiones vitalicias, pero en el Ecuador las pensiones vitalicias de los ex mandatarios no son hasta el final de la vida de ellos ya que dicha pensión podrá ser heredada por su cónyuge, conviviente o hijos, caso contrario a lo que sucede con un servidor público normal, el cual si fallece, dicha pensión que recibía por sus años de trabajo no se heredara a ningún familiar, así mismo, "Las pensiones, hacen referencia a las cantidades monetarias que una persona recibe de forma regular o vitalicia, de una institución u organismo, en este caso del Estado, por motivo de cumplir con los requisitos necesarios para su goce" (SisalimaSalinas, 2019, p. 40). 
De modo que, para hablar sobre la pensión vitalicia es necesario mencionar que se han presentado varias propuestas de proyectos para reformar la Ley Orgánica de Servicio Público, pues se determinó el inconformismo de que algunos familiares de ex mandatarios reciben la pensión vitalicia, como es el caso de la hija de Jamil Mahuad, la actual esposa del ex presidente Abdalá Bucaram y la hermana del ex presidente Rafael Correa. También una de las propuestas de reformas que se presentó ante la Comisión de Derecho de los Trabajadores, es la existencia de ex mandatarios que no terminaron su periodo por circunstancias de carácter político, pero siguen recibiendo su pensión, como es el caso de Fabián Alarcón, Rosalía Arteaga, Lucio Gutiérrez y Alfredo Palacios. (campoverde, 2019).

Las pensiones vitalicias son de carácter unipersonal, por lo consiguiente al ser cobradas por familiares o terceros estando el titular de este beneficio con vida; atentan con lo establecido en la Ley que regula esta figura jurídica, tal como lo establece (SisalimaSalinas, 2019), al expresar que tanto la señora Rosa Pulley delegada de Abdalá Bucaram, Paola Mahuad delegada por Jamil Mahuad, Pierina Correa delegada por Rafael Correa gozan de este beneficio transgrediendo lo dispuesto por la Ley Orgánica de Servicio Público.

Por otra parte, ex presidentes que no han terminado su periodo de mandato siguen siendo beneficiarios de la pensión vitalicia, pese a que ellos desarrollaron acciones que generaron perjuicios al Estado, como por ejemplo peculado, enriquecimiento ilícito, cohecho. Por todas estas situaciones cabe el estudio del pago de la pensión vitalicia a todos los ex mandatarios constitucionales del Ecuador.

Un ejemplo claro de que la figura de la pensión vitalicia es aplicada de manera incorrecta, se ve reflejada en lo que establecen los autores (Tuchie \& Espinel, 2016), sobre el caso del ex presidente del Ecuador Jamil Mahuad, quien aun siendo derrocado y apartado de sus funciones como cabeza de estado a causa de la mala administración pública desarrollada por este, que acarreo consecuencias como un exceso de inflación dentro del estado o el paro de la mitad de entidades financieras del país. Aun con estos 
antecedentes este ex mandatario es beneficiario de la pensión vitalicia, a pesar que la Ley establezca que este no debería percibirla.

Se habla de herencia a todo derecho u obligación que es transmitida por una persona a otra al momento de su muerte, convirtiéndose estos en herederos (Rodríguez, 2017), por otro lado, la (Ley Orgánica de Servicio Público, 2010), establece que el derecho de pensión vitalicia será hereditable pudiendo beneficiar a su cónyuge o conviviente en unión de hecho en caso de fallecer el titular de este beneficio, se les reconocerá a los hijos del titular con la condicionante de que sean menores de edad y mayores con algún tipo de discapacidad grave en caso de faltar en cónyuge.

Sin embargo, actualmente recibe la pensión vitalicia los hijos mayores de edad del fallecido ex presidente Jaime Roldós Aguilera sin tener ningún tipo de discapacidad grave o ser menores de edad, a pesar que la Ley Orgánica de Servicio Público es clara al especificar las condicionantes que deben tener los hijos para percibir este beneficio. (González, 2020).

Por otro lado, (Quizhpe-Benítez, 2019), considera que las pensiones vitalicias deberían ser cobradas solo para los ex presidentes y ex vicepresidentes y no ser heredadas a sus familiares; ya que fueron ellos los que trabajaron por el país, además hace referencia de quienes no hayan culminado los 4 años de su mandato no deberían premiarlos con un sueldo vitalicio. Por ello, (Sisalima-Salinas, 2019), establece como una de sus propuestas eliminar del marco jurídico ecuatoriano que la pensión vitalicia sea heredada para el cónyuge o conviviente de ex mandatarios constitucionales del Ecuador.

En este sentido, la investigación tiene por objetivo analizar la pensión vitalicia y su vigencia desde la ley orgánica de servicio público en el territorio ecuatoriano. 


\section{MÉTODO}

La investigación se basó desde una centralidad metodológica en un tipo descriptiva documental con diseño bibliográfico, lo cual permitió recopilar una población de artículos de investigación, entrevistas en diarios digitales, leyes; lo cual permitió desde el método analítico - sintético construir una síntesis teórica como propuesta investigativa, empleándose para tal fin, la técnica de análisis de contenido jurídico como elemento cooperador a los investigadores para estructurar el proceso investigativo.

\section{RESULTADOS}

En función de los documentos escrutados, se presentan los siguientes resultados:

\section{Ex presidentes y Ex vicepresidentes del Ecuador que reciben pensión vitalicia}

El Ecuador gasta por concepto de pago de pensiones vitalicias un total de 979,665 dólares, a causa de esto el presidente Lenin Moreno presentó el decreto ejecutivo Nro.135, el cual fue emitido por la necesidad de austeridad en el gasto de la administración pública y de esta manera garantizar la correcta ejecución de los recursos públicos reduciendo los salarios de los servidores públicos, pero sin tocar las pensiones vitalicias transgrediendo de esta manera el principio de igualdad, al no reconocer a los beneficiarios de la pensión vitalicia como servidores públicos. (Brito-Zhañay \& PalaciosVintimilla, 2021).

De conformidad con lo que establece la (Ley Organica de Servicio Público, 2010), los ex mandatarios que reciben un sueldo vitalicio después de su mandato percibirán un $75 \%$ de su última remuneración, siempre que hayan sido elegidos por votación popular y posesionados de su cargo, así mismo, el autor (Jerez-Jimenez, 2018), establece que a lo largo del tiempo la pension vitalicia prevista para ex mandatarios constitucionales derrocados de su periodo de mandato ha ido incrementando su valor exponencialmente, creando de esta manera desventaja económica en la ciudadania al aumentar los impuestos y tamien ocasionar en cierta medida una perdida de la pension económica a 
los ciudadanos.

En las siguientes tablas se especifica los nombres de los ex presidentes y ex vicepresidentes que son beneficiarios de la pensión vitalicia y el valor que cobran de manera mensual:

\section{Tabla 1.}

Tabla de ex presidentes que reciben pensión vitalicia.

\section{EX PRESIDENTES}

\begin{tabular}{ll}
\hline Rodrigo Borja & $\$ 4.226,25$ \\
Abdalá Bucaram & $\$ 4.226,25$ \\
Oswaldo Hurtado & $\$ 4.226,25$ \\
Sixto Durán Ballén & $\$ 4.226,25$ \\
Jamil Mahuad & $\$ 4.226,25$ \\
Gustavo Noboa & $\$ 4.226,25$ \\
Lucio Gutiérrez & $\$ 4.226,25$ \\
Alfredo Palacio & $\$ 4.226,25$ \\
Rafael Correa & $\$ 4.226,25$ \\
\hline Fabián Alarcón & $\$ 3.200,00$ \\
\hline
\end{tabular}

Fuente: Elaboración propia a partir de Gomez-Ponce (2018). 
Tabla 2.

Tabla de ex presidentes que reciben pensión vitalicia.

\begin{tabular}{lc}
\hline EX VICEPRESIDENTES & PENSIÓN ACTUAL \\
\hline Blasco Peñaherrera & $\$ 4.057,50$ \\
Luis Parodi & $\$ 4.057,50$ \\
Rosalía Arteaga & $\$ 4.057,50$ \\
Lenin Moreno & $\$ 4.057,50$ \\
Alberto Dahik & $\$ 4.057,50$ \\
León Roldós & $\$ 2.400,00$ \\
Jorge Glas & $\$ 4.057,50$ \\
Eduardo Peña & $\$ 2.400,00$ \\
Pedro Aguayo & $\$ 2.400,00$ \\
Pedro Pinto & $\$ 2.400,00$ \\
\hline Alejandro Serrano & $\$ 2.400,00$ \\
\hline
\end{tabular}

Fuente: Elaboración propia a partir de Gomez-Ponce (2018).

El presidente del Ecuador Lenin Moreno desarrolló la Ley de Apoyo Humanitario con el objetivo de afrontar la crisis a causa de la pandemia COVID - 19, mediante esta se aplicó una reforma jurídica a la Ley de Servicio Público, en la cual se incrementaron dos causales con respecto a la pensión vitalicia. (Coba, 2020).

Las causales establecidas en la Ley de Apoyo Humanitario mediante la cual se desarrollaron reformas a la pensión vitalicia exceptuaron al pago de esta a los ex mandatarios que no hayan concluido el período para el que fueron electos y también a quienes hayan sido sentenciados por delitos contra la administración pública y delitos que atentan contra la vida y de lesa humanidad. (Garcia-Ruiz \& Guevara-Arcentales, 2020). Con base a lo que estableció la (Ley de Apoyo Humanitario, 2020), en referencia a los beneficiarios pension vitalicia se vetó el pago de este beneficio a los ex mandatarios 
Rafael Correa Delgado y Jorge Glas Espinel, al haber sido sentenciados por la justicia ecuatoriana como culpables del caso sobornos.

\section{La pensión vitalicia y su incidencia en el presupuesto general del estado}

De acuerdo los registros emitidos por el Ministerio de Finanzas al Observatorio de Gasto Público de la Fundación Ciudadanía y Desarrollo en el 2008 se destinaba para el pago de la pensión vitalicia un total de USD 595.000, sin embargo, esta cifra aumentó a poco más de 1'084.000, al cierre de 2018. (Gomez-Ponce, 2018). En base a lo que expresa el autor en un lapso de 10 años el presupuesto destinado a pensiones vitalicias ha doblado su valor, haciendo más fuerte la carga de estas pensiones para el presupuesto general del Estado, hecho que si se sigue desarrollado de la misma manera podría representar una gran carga económica en los siguientes años para el Ecuador.

Considerando la difícil situación en la que el Ecuador no se encuentra en este momento económicamente bien, no es aceptable que se continúe financiando estas pensiones vitalicias elevadas a los ex mandatarios, ya que se destina diariamente cerca de $\$ 2.700$ dólares equivalente a un valor de $\$ 81.538,75$ mensual para el pago de este beneficio, cuando sectores como la salud y la educación han sufrido recortes presupuestarios en los últimos años (Brito-Zhañay \& Palacios-Vintimilla, 2021).

El decreto emitido por el presidente Lenin Moreno establece reducir el 10\% de los sueldos que se paga a los servidores públicos con el único fin de optimizar los recursos del Estado, a raíz de aquello el autor (Quizhpe-Benítez, 2019), considera que se debe eliminar la pensión vitalicia a ex mandatarios que no son merecedores de este beneficio, considerando esto necesario porque se está haciendo un mal gasto del presupuesto del Estado.

Sin embargo, la autora (Mejia-Toapanta, 2020), expresa que la falta de un manejo correcto de la figura juridica de pensión vitalicia hace insostenible su existencia debido a las dificultades economicas que atravisa el Ecuador en estos momentos, desarrollando una situacion de crisis y problemática sociales en lo referente a las garantias de derechos 
que tiene todo ciudadano ecuatoriano.

Desde las diferentes opiniones de los autores acerca de la incidencia que tiene el pago de la pensión vitalicia en el presupuesto general del Estado, se llegó a determinar que el Ecuador invierte una gran cantidad al pago de los ex mandatarios, considerando que se exceptúa al pago de la pensión vitalicia para ex mandatarios que fueron derrocados, también una vez fallecido el titular de este beneficio serán heredadas, a pesar de ello en la actualidad existen ex mandatarios constitucionales derrocados y otros que han delegado el pago de su pensión vitalicia; por eso se establece que no se aplica correctamente lo establecido en la ley y se sigue reconociendo beneficios a quienes no son merecedores, obteniendo como desventaja más gastos para el Ecuador.

\section{Vulneración de derechos constitucionales}

La Constitución del Ecuador establece que el Ecuador es un estado constitucional de derecho y justicia, cuya soberanía radica en los ciudadanos y debe cumplirse por los diferentes órganos del poder público. (Garcia-Ruiz \& Guevara-Arcentales, 2020). En la regulación de la pensión vitalicia, no se aplica el deber primordial del estado, como está prescrito en el artículo 3 de la (Constitución de la República del Ecuador, 2008), la cual es clara al establecer que el Estado Ecuatoriano velará por el desarrollo nacional, combatirá la pobreza y realizará una correcta redistribución de los recursos y la riqueza para acceder al buen vivir.

En consideración del articulo antes mencionado, cabe recalcar que el dinero que el Estado invierte mensual en ex mandatarios es de 81.538,75 dólares, los cuales se reparten a ex gobernantes que llegaron a esa posición de diferente manera, unos culminando correctamente con su periodo, otros dejándolo a medias, también a quienes cometieron delitos que atentan contra la administración pública; es decir todos son merecedores de este derecho sin importar como terminaron su periodo gubernamental, cuando estos deberían ser separados en merecedores y no merecedores, por todo ello en relación a la problemática expuesta, no deberían ser beneficiarios de esta pensión 
vitalicia los que no desempeñaron su función de contribuir al país, y por ello se debería vetar la pensión vitalicia a estas personas, pudiendo invertir estos recursos recuperados en sectores afectados por los recortes presupuestarios; tales como salud, educación, arte, sectores beneficiosos que son necesarios para el desarrollo social del país.

\section{DISCUSIÓN}

El autor (Quizhpe-Benítez, 2019), expresa la idea de la necesidad de reforma que debe producirse en la Ley Orgánica de Servicio Público con respecto a la figura jurídica de la pensión vitalicia, estableciendo requisitos para poder ser merecedor de este beneficio y de la misma manera implementar causales para la perdida de este derecho.

Mediante los resultados obtenidos de la investigación del presente objeto de estudio, se puede evidenciar la problemática de acuerdo con beneficiar al pago de las pensiones vitalicias a quienes no han terminado todo su periodo de mandato, como también para quienes hayan cometido delitos que atentan contra la administración pública, como se puede observar la Ley Orgánica de Servicio Público, no se encuentra limitada en relación a la pensión vitalicia, ni argumentada de manera explícita al no establecer preceptos legales claros de quienes pueden ser o no beneficiarios de la pensión vitalicia y de esta manera no afectar en partes al desarrollo económico del país.

Los autores (Brito-Zhañay \& Palacios-Vintimilla, 2021), coinciden en que la figura de la pension vitalicia para ex mandatarios constitucionales es perjudicial para el estado euatoriano, considerando que en la epoca que se creó este beneficio no es la misma por la que esta pasando el pais actualmente, por ello cabe una reforma a la estructura juridica de la pension vitalicia.

En concordancia con lo establecido es claro que la figura de la pensión vitalicia no está completamente desarrollada en la Ley Orgánica de Servicio Público, pues en el estado actual de la figura jurídica el estado ecuatoriano asume el pago de pensiones vitalicias a ex mandatarios que no culminaron su periodo, que cometieron delitos que atentan contra la administración pública o a terceras personas representantes de ex mandatarios. Por 
todos estos acontecimientos es notoria la concordancia con varios autores que cuestionan y critican esta figura jurídica, reclamando una reforma completa para que este beneficio sea asignado merecidamente.

\section{CONCLUSIONES}

Desde la fundamentación teórica expuesta se logra concluir que, la pensión vitalicia es un derecho asignado a los ex mandatarios constitucionales del Ecuador, el cual consta de un pago vitalicio del 75 por ciento del sueldo que percibían durante su mandato, regulado por los artículos 135 y 136 de la Ley Orgánica de servicio público 2010, presentando notables falencias en su estructura jurídica, al no regular completamente, esta figura jurídica, estableciendo características que deberían ser cumplidas por los ex mandatarios, tales como haber cumplido su periodo, no haber tenido problemas con la justicia, o una limitación concreta en la herencia de la pensión vitalicia, tal como se ha presentado en los sinnúmeros de proyectos de reforma a esta figura jurídica.

Desde el diagnóstico del estado actual de la figura jurídica de la pensión vitalicia, con la metodología empleada, así como con los métodos de investigación se logra determinar que en la actualidad el estado ecuatoriano dedica más de un millón de dólares del presupuesto general del estado en pago de pensiones vitalicias a ex mandatarios constitucionales. La problemática que se desarrolla ante este suceso, es que, en un porcentaje alto existen terceras personas cobrando este beneficio, personas que no deberían tener el derecho de obtener este beneficio, como son los casos de Pierina Correa o de hijos de ex mandatarios, que sin ser menores de edad o sin tener ningún tipo de discapacidad grave, disfrutan del pago de este beneficio, transgrediendo totalmente lo establecido por la Ley orgánica de Servicio Público.

Desde el análisis jurídico realizado sobre la pensión vitalicia para ex mandatarios del Ecuador, se determina que, la figura jurídica de la Pensión vitalicia, necesita una reforma completa, puesto que al estar normada en tan solo dos artículos, estos norman esta figura jurídica de manera general, dando pie, a que familiares o terceros de ex mandatarios, 
asuman este beneficio en su nombre, desarrollando un perjuicio a la economía ecuatoriana, por lo tanto, es necesario limitar este pago a máximo 10 años y cambiar la figura jurídica de pensión vitalicia a pago de reconocimiento a ex presidentes y ex vicepresidentes constitucionales.

\section{FINANCIAMIENTO}

No monetario.

\section{AGRADECIMIENTO}

A la Universidad Regional Autónoma de los Andes, Quevedo; por motivar el desarrollo de la Investigación.

\section{REFERENCIAS CONSULTADAS}

Brito-Zhañay, J. M., \& Palacios-Vintimilla, C. P. (2021). Eliminación de pensiones vitalicias a ex Presidentes y Vicepresidentes que han desarrollado sus funciones sujetas a la corrupción y destitución [Elimination of life pensions for former Presidents and Vice Presidents who have performed their functions subject to corruption and dismissal]. FIPCAEC, 378-401.

Campoverde, J. P. (2019). Pensiones vitalicias son cobradas por familiares de exmandatarios [Life pensions are collected by relatives of ex-presidents]. Obtenido de https://n9.cl/15yi7

Coba, G. (2020). Ley Humanitaria: veto parcial del Ejecutivo hace 32 observaciones [Humanitarian Law: partial veto of the Executive makes 32 observations]. Recuperado de https://n9.cl/k96zt

Constitución de la República del Ecuador. Registro Oficial 449 de 20-oct-2008. https://n9.cl/hd0q

Garcia-Ruiz, I. E., \& Guevara-Arcentales, M. J. (2020). Análisis del vacío normativo vigente en torno a la pensión vitalicia para ex mandatarios en el Ecuador [Analysis of the current regulatory gap around the life pension for former presidents in Ecuador]. Universidad Católica de Santiago de Guayaquil. http://repositorio.ucsg.edu.ec/handle/3317/15754 
Gomez-Ponce, L. (2018). Pensiones vitalicias de ex mandatarios en debate [Life pensions of former leaders in debate]. Recuperado de https://n9.cl/zdk9

González, D. (2020). Sueldos vitalicios: un dilema de moralidad [Life wages: a morality dilemma]. Recuperado de https://n9.cl/jseb8

Jerez-Jimenez, T. E. (2018). El pago de la pensión vitalicia mensuala favor de las y los señores ex presidentes y vicepresidentes constitucionales de la república del ecuador derrocados, vulnerando el patrimonio del Estado [The payment of the monthly lifetime pension in favor of the former constitutional presidents and vicepresidents of the republic of Ecuador overthrown, violating the State's patrimony]. UNIANDES Ambato. http://dspace.uniandes.edu.ec/handle/123456789/9004

Ley de Apoyo Humanitario. Registro Oficial Suplemento 229 de 22-jun.-2020. https://n9.cl/8yi7

Ley Orgánica de Servicio Público. Registro Oficial № 294. Miércoles 6 de Octubre del 2010. https://n9.cl/do7v7

Mejia-Toapanta, A. M. (2020). Las pensiones vitalicias de ex mandatarios constitucionales [Life pensions of former constitutional leaders]. UNIANDES IBARRA. http://dspace.uniandes.edu.ec/handle/123456789/11142

Quizhpe-Benítez, E. S. (2019). Eliminación de la pensión vitalicia a los ex presidentes y ex vicepresidentes,que no terminen su periodo de mandato y los que incurran en delitos relacionados a sus funciones de gobierno [Elimination of the life pension for former presidents and former vice presidents, who do not complete their term of office and those who commit crimes related to their government functions]. Univerisdad Nacional de Loja. https://n9.cl/0n3mu

Rodríguez Tapia, C. G. (2017). Problemática jurídica de las herencias, donaciones y legados, como objetos de imposición [Legal problems of inheritances, donations and legacies, as objects of taxation]. Recuperado de https://n9.cl/pxizf

Sisalima-Salinas, F. S. (2019). La pensión vitalicia para el cónyuge o conviviente de ex presidentes y vicepresidentes constitucionales de la república del Ecuador en el caso de su fallecimiento es innecesaria [The life pension for the spouse or partner of former constitutional presidents and vice presidents of the Republic of Ecuador in the event of their death is unnecessary]. Univerisdad Nacional de Loja. http://dspace.unl.edu.ec/jspui/handle/123456789/22772 
Iustitia Socialis. Revista Arbitrada de Ciencias Jurídicas.

$$
\text { Año VI. Vol. VI. N }{ }^{\circ} \text { 10. Enero - Junio. } 2021
$$

Hecho el depósito de Ley: FA2016000064

ISSN: 2542-3371

FUNDACIÓN KOINONIA (F.K). Santa Ana de Coro, Venezuela

Rosa María Suárez-Barzola; Wilson Eduardo Castro-Núñez; Pamilys Milagros Moreno-Arvelo

Tuchie, V., \& Espinel, K. (2016). Presidentes derrocados del Ecuador [Overthrown Presidents of Ecuador]. https://n9.cl/covl7

(C2021 por los autores. Este artículo es de acceso abierto y distribuido según los términos y condiciones de la licencia Creative Commons Atribución-NoComercial-Compartirlgual 4.0 Internacional (CC BY-NC-SA 4.0)

(https://creativecommons.org/licenses/by-nc-sa/4.0/). 